\title{
Boundary Control for Cooperative Elliptic Systems under Conjugation Conditions
}

\author{
H. M. Serag1 , L. M. Abd-Elrhman², A. A. Alsaban³ \\ ${ }^{1}$ Department of Mathematics, Faculty of Science, Al-Azhar University, Nasr City, Cairo, Egypt \\ ${ }^{2}$ Department of Mathematics, Faculty of Science, Al-Azhar University [for Girls], Nasr City, Cairo, Egypt \\ ${ }^{3}$ Department of Mathematics, Faculty of Science, Ibb University, Ibb, Yemen \\ Email: serraghm@yahoo.com, lamia.abdelrhman3461@yahoo.com, ahmedalsaban@yahoo.com
}

How to cite this paper: Serag, H.M., AbdElrhman, L.M. and Alsaban, A.A. (2021) Boundary Control for Cooperative Elliptic Systems under Conjugation Conditions. Advances in Pure Mathematics, 11, 457-471. https://doi.org/10.4236/apm.2021.115032

Received: February 3, 2021

Accepted: May 22, 2021

Published: May 25, 2021

\section{Copyright (c) 2021 by author(s) and} Scientific Research Publishing Inc. This work is licensed under the Creative Commons Attribution International License (CC BY 4.0).

http://creativecommons.org/licenses/by/4.0/

\begin{abstract}
The existence and uniqueness of the state for $2 \times 2$ Dirichlet cooperative elliptic systems under conjugation conditions are proved using Lax-Milgram lemma, then the boundary control for these systems is discussed. The set of equations and inequalities that characterizes this boundary control is found by theory of Lions, Sergienko and Deineka. The problem for cooperative Neumann elliptic systems under conjugation conditions is also considered. Finally, the problem for $n \times n$ cooperative elliptic systems under conjugation conditions is established.
\end{abstract}

\section{Keywords}

Cooperative Systems, Conjugation Conditions, Dirichlet and Neumann Conditions, Existence and Uniqueness of Solutions, Boundary Control

\section{Introduction}

In today's rapidly progressing science and technology, the field of control theory is at the forefront of the creative interplay of mathematics, engineering, and computer science.

Control theory has two objectives:

To understand the fundamental principle of control, and to characterize them mathematically.

The control problem is to choose the control space $U$ to minimize an energy functional $Л(u)$, subject to constraints on the control such as $U_{a d}$ (set of admissible control) is a closed convex subset of $U$.

Various optimal control problems, of systems governed by finite order elliptic, parabolic and hyperbolic operators with finite number of variables have been in- 
troduced by Lions [1]. These problems have been extended to non-cooperative systems in [2] [3] and cooperative systems in [4] [5] [6] [7]. The control problems for infinite order hyperbolic operators have been studied in [8] [9].

Some existence results have been established for nonlinear systems in [10] [11] [12] [13] [14].

Some applications for control problems were introduced for example in [2] [15].

New optimal control problems of distributed systems described by an elliptic, parabolic and hyperbolic operators with conjugation conditions and by a quadratic cost functional have been studied by Sergienko and Deineka [16] [17] [18].

In the present work, using the theory of Lions [1], Sergienko and Deineka [16] [17] [18], the boundary control for some cooperative elliptic systems of the form

$$
-\Delta h_{i}=\sum_{j=1}^{n} a_{i j} h_{j}+f_{i} \quad \text { in } \Omega, i, j=1,2,3, \cdots, n,
$$

under conjugation conditions is discussed, where

$$
a_{i j}>0 \quad \forall i \neq j .
$$

System (1), where (2) is satisfied is called cooperative system. Such systems appear in some biological and physical problems [19].

Our paper is organized as follows: In Section 2, we first prove the existence and uniqueness of the state for $2 \times 2$ Dirichlet cooperative system under conjugation conditions, then we study the optimal boundary control of this system. Section 3 is devoted to discuss the boundary control for $2 \times 2$ Neumann cooperative elliptic system under conjugation conditions. In Section 4, we generalize the discussion which has been introduced in Section 2, to $n \times n$ Dirichlet cooperative system with conjugation conditions. Finally in Section 5 , we generalize the problem which has been established in Section 3 to $n \times n$ Neumann cooperative elliptic system under conjugation conditions.

\section{Boundary Control for $2 \times 2$ Dirichlet Elliptic Systems}

In this section, we study the boundary control for the following $2 \times 2$ cooperative Dirichlet elliptic system

$$
\begin{cases}-\Delta h_{1}=a h_{1}+b h_{2}+f_{1} & \text { in } \Omega, \\ -\Delta h_{2}=c h_{1}+d h_{2}+f_{2} & \text { in } \Omega, \\ h_{1}=h_{2}=0 & \text { on } \Gamma,\end{cases}
$$

under conjugation conditions:

$$
\begin{cases}R_{1}\left\{\frac{\partial h_{1}}{\partial v_{A}}\right\}^{-}+R_{2}\left\{\frac{\partial h_{1}}{\partial v_{A}}\right\}^{+}=\left[h_{1}\right]+\delta & \text { on } \gamma, \\ R_{1}\left\{\frac{\partial h_{2}}{\partial v_{A}}\right\}^{-}+R_{2}\left\{\frac{\partial h_{2}}{\partial v_{A}}\right\}^{+}=\left[h_{2}\right]+\delta & \text { on } \gamma,\end{cases}
$$




$$
\left\{\begin{array}{l}
{\left[\frac{\partial h_{1}}{\partial v_{A}}\right]=\left[\sum_{i, j=1}^{n} \frac{\partial h_{1}}{\partial x_{j}} \cos \left(v, x_{i}\right)\right]=w_{1} \quad \text { on } \gamma,} \\
{\left[\frac{\partial h_{2}}{\partial v_{A}}\right]=\left[\sum_{i, j=1}^{n} \frac{\partial h_{2}}{\partial x_{j}} \cos \left(v, x_{i}\right)\right]=w_{2} \quad \text { on } \gamma,}
\end{array}\right.
$$

where

$a, b, c$ and $d$ are given numbers such that $b, c>0$,

and

$$
R_{1}, R_{2}, w, \delta \in C(\gamma), R_{1}, R_{2} \geq 0, R_{1}+R_{2} \geq R_{0}>0, R_{0}=\text { constant. }
$$

We first prove the existence of the state of systems (3) under the following conditions:

$$
\left\{\begin{array}{l}
a<\mu, d<\mu, \\
(\mu-a)(\mu-d)>b c
\end{array}\right.
$$

where $\mu$ is a positive constant determined by Friedrich inequality:

$$
\mu \int_{\Omega}|h|^{2} \mathrm{~d} x \leq \int_{\Omega}|\nabla h|^{2} \mathrm{~d} x .
$$

Then, we prove the existence of boundary control for this system and we find the set of equations and inequalities that characterizes this boundary control.

Existence and uniqueness of the state

By Cartesian product, we have the following chain of Sobolev spaces:

$$
\left(H_{0}^{1}(\Omega)\right)^{2} \subseteq\left(L^{2}(\Omega)\right)^{2} \subseteq\left(H^{-1}(\Omega)\right)^{2} .
$$

On $\left(H_{0}^{1}(\Omega)\right)^{2}$, we define the bilinear form:

$$
\begin{aligned}
a(h, \psi)= & \int_{\Omega} \nabla h_{1} \nabla \psi_{1} \mathrm{~d} x+\int_{\Omega} \nabla h_{2} \nabla \psi_{2} \mathrm{~d} x \\
& -\int_{\Omega}\left(a h_{1} \psi_{1}+b h_{2} \psi_{1}+c h_{1} \psi_{2}+d h_{2} \psi_{2}\right) \mathrm{d} x \\
& +\int_{\gamma} \frac{\left[h_{1}\right]\left[\psi_{1}\right]}{R_{1}+R_{2}} \mathrm{~d} \gamma+\int_{\gamma} \frac{\left[h_{2}\right]\left[\psi_{2}\right]}{R_{1}+R_{2}} \mathrm{~d} \gamma .
\end{aligned}
$$

Then, we have

Lemma 2.1 The bilinear form (9) is coercive on $\left(H_{0}^{1}(\Omega)\right)^{2}$; that is, there exists a positive constant $C$ such that

$$
\left.a(h, h) \geq C\|h\|_{\left[H_{0}^{1}(\Omega)\right.}^{2}\right]^{2} \quad \forall h=\left\{h_{1}, h_{2}\right\} \in\left(H_{0}^{1}(\Omega)\right)^{2} .
$$

Proof.

As in [19], we choose $m$ is large enough such that $a+m>0$ and $d+m>0$.

Then,

$$
\begin{aligned}
a(h, h)= & \frac{1}{b} \int_{\Omega}\left(\left|\nabla h_{1}\right|^{2}+m\left|h_{1}\right|^{2}\right) \mathrm{d} x+\frac{1}{c} \int_{\Omega}\left(\left|\nabla h_{2}\right|^{2}+m\left|h_{2}\right|^{2}\right) \mathrm{d} x \\
& -\frac{a+m}{b} \int_{\Omega}\left|h_{1}\right|^{2} \mathrm{~d} x-\frac{d+m}{c} \int_{\Omega}\left|h_{2}\right|^{2} \mathrm{~d} x-2 \int_{\Omega} h_{1} h_{2} \mathrm{~d} x \\
& +\int_{\gamma} \frac{\left[h_{1}\right]\left[\psi_{1}\right]}{R_{1}+R_{2}} \mathrm{~d} \gamma+\int_{\gamma} \frac{\left[h_{2}\right]\left[\psi_{2}\right]}{R_{1}+R_{2}} \mathrm{~d} \gamma .
\end{aligned}
$$


From (6), we get

$$
\begin{aligned}
a(h, h) \geq & \frac{1}{b} \int_{\Omega}\left(\left|\nabla h_{1}\right|^{2}+m\left|h_{1}\right|^{2}\right) \mathrm{d} x+\frac{1}{c} \int_{\Omega}\left(\left|\nabla h_{2}\right|^{2}+m\left|h_{2}\right|^{2}\right) \mathrm{d} x \\
& -\frac{a+m}{b} \int_{\Omega}\left|h_{1}\right|^{2} \mathrm{~d} x-\frac{d+m}{c} \int_{\Omega}\left|h_{2}\right|^{2} \mathrm{~d} x-2 \int_{\Omega} h_{1} h_{2} \mathrm{~d} x .
\end{aligned}
$$

By Cauchy Schwartz inequality

$$
\begin{aligned}
a(h, h) \geq & \frac{1}{b} \int_{\Omega}\left(\left|\nabla h_{1}\right|^{2}+m\left|h_{1}\right|^{2}\right) \mathrm{d} x+\frac{1}{c} \int_{\Omega}\left(\left|\nabla h_{2}\right|^{2}+m\left|h_{2}\right|^{2}\right) \mathrm{d} x \\
& -\frac{a+m}{b} \int_{\Omega}\left|h_{1}\right|^{2} \mathrm{~d} x-\frac{d+m}{c} \int_{\Omega}\left|h_{2}\right|^{2} \mathrm{~d} x \\
& -2\left(\int_{\Omega}\left|h_{1}\right|^{2} \mathrm{~d} x\right)^{\frac{1}{2}}\left(\int_{\Omega}\left|h_{2}\right|^{2} \mathrm{~d} x\right)^{\frac{1}{2}} .
\end{aligned}
$$

From (8), we deduce

$$
a(h, h) \geq \frac{1}{b}\left(1-\frac{a+m}{\mu+m}\right)\left\|h_{1}\right\|^{2}+\frac{1}{c}\left(1-\frac{d+m}{\mu+m}\right)\left\|h_{2}\right\|^{2}-\frac{2}{\mu+m}\left\|h_{1}\right\| h_{2} \| .
$$

Therefore (7) implies

$$
\left.a(h, h) \geq C\left(\left\|h_{1}\right\|^{2}+\left\|h_{2}\right\|^{2}\right)=C\|h\|_{\left[H_{0}^{1}(\Omega)\right.}^{2}\right]^{2} \quad \forall h \in\left(H_{0}^{1}(\Omega)\right)^{2},
$$

which proves the coerciveness condition of the bilinear form (2.7). Then using Lax-Milgram lemma, we can prove the following theorem:

Theorem 2.1 For a given $f=\left(f_{1}, f_{2}\right) \in\left(L^{2}(\Omega)\right)^{2}$ there exists a unique solution $h=\left(h_{1}, h_{2}\right) \in\left(H_{0}^{1}(\Omega)\right)^{2}$ for systems (3) with conjugation conditions (4) and (5) if conditions (7) are satisfied.

\section{Formulation of the control problem}

The space $U=\left(L^{2}(\Gamma)\right)^{2}$ is the space of controls. For a control $u=\left\{u_{1}, u_{2}\right\} \in\left(L^{2}(\Gamma)\right)^{2}$, the state $h(u)=\left\{h_{1}(u), h_{2}(u)\right\}$ of the system is given by the solution of

$$
\begin{cases}-\Delta h_{1}(u)=a h_{1}(u)+b h_{2}(u)+f_{1} & \text { in } \Omega, \\ -\Delta h_{2}(u)=c h_{1}(u)+d h_{2}(u)+f_{2} & \text { in } \Omega, \\ h_{1}(u)=u_{1}, h_{2}(u)=u_{2} & \text { on } \Gamma,\end{cases}
$$

under conjugation conditions

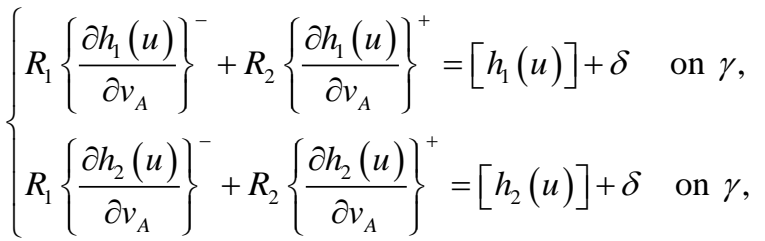

$$
\begin{aligned}
& \left\{\begin{array}{l}
{\left[\frac{\partial h_{1}(u)}{\partial v_{A}}\right]=\left[\sum_{i, j=1}^{n} \frac{\partial h_{1}(u)}{\partial x_{j}} \cos \left(v, x_{i}\right)\right]=w_{1} \quad \text { on } \gamma,} \\
{\left[\frac{\partial h_{2}(u)}{\partial v_{A}}\right]=\left[\sum_{i, j=1}^{n} \frac{\partial h_{2}(u)}{\partial x_{j}} \cos \left(v, x_{i}\right)\right]=w_{2} \quad \text { on } \gamma .}
\end{array}\right.
\end{aligned}
$$


The observation equation is given by:

$$
z(u)=\left\{z_{1}(u), z_{2}(u)\right\}=C h(u)=C\left\{h_{1}(u), h_{2}(u)\right\}=\left\{h_{1}(u), h_{2}(u)\right\}
$$

For a given $z_{d}=\left\{z_{1 d}, z_{2 d}\right\} \in\left(L^{2}(\Gamma)\right)^{2}$, the cost functional is given by

$$
J(v)=\left\|\frac{\partial h_{1}(v)}{\partial v_{A}}-z_{1 d}\right\|_{L^{2}(\Gamma)}^{2}+\left\|\frac{\partial h_{2}(v)}{\partial v_{A}}-z_{2 d}\right\|_{L^{2}(\Gamma)}^{2}+(N v, v)_{\left(L^{2}(\Gamma)\right)^{2}},
$$

where $N$ is a hermitian positive definite operator such that:

$$
(N v, v)_{\left(L^{2}(\Gamma)\right)^{2}} \geq Q\|v\|_{\left(L^{2}(\Gamma)\right)^{2}}^{2}, \quad Q>0 .
$$

The control problem then is to:

$$
\left\{\begin{array}{l}
\text { Find } u=\left\{u_{1}, u_{2}\right\} \in U_{a d}\left(\text { closed convex subset of }\left(L^{2}(\Gamma)\right)^{2}\right) \text { such that : } \\
J(u)=\inf J(v) \quad \forall v \in U_{a d} .
\end{array}\right.
$$

The cost functional (14) can be written as

$$
\begin{aligned}
J(v)= & \left\|\frac{\partial h_{1}(v)}{\partial v_{A}}-\frac{\partial h_{1}(0)}{\partial v_{A}}+\frac{\partial h_{1}(0)}{\partial v_{A}}-z_{1 d}\right\|_{L^{2}(\Gamma)}^{2} \\
& +\left\|\frac{\partial h_{2}(v)}{\partial v_{A}}-\frac{\partial h_{2}(0)}{\partial v_{A}}+\frac{\partial h_{2}(0)}{\partial v_{A}}-z_{2 d}\right\|_{L^{2}(\Gamma)}^{2} \\
& +(N v, v)_{\left(L^{2}(\Gamma)\right)^{2}} .
\end{aligned}
$$

If we let:

$$
\begin{aligned}
\pi(u, v)= & \left(\frac{\partial h_{1}(u)}{\partial v_{A}}-\frac{\partial h_{1}(0)}{\partial v_{A}}, \frac{\partial h_{1}(v)}{\partial v_{A}}-\frac{\partial h_{1}(0)}{\partial v_{A}}\right)_{L^{2}(\Gamma)} \\
& +\left(\frac{\partial h_{2}(u)}{\partial v_{A}}-\frac{\partial h_{2}(0)}{\partial v_{A}}, \frac{\partial h_{2}(v)}{\partial v_{A}}-\frac{\partial h_{2}(0)}{\partial v_{A}}\right)_{L^{2}(\Gamma)} \\
& +(N u, v)_{\left(L^{2}(\Gamma)\right)^{2}} \cdot
\end{aligned}
$$

and

$$
\begin{aligned}
f(v)= & \left(z_{1 d}-\frac{\partial h_{1}(0)}{\partial v_{A}}, \frac{\partial h_{1}(v)}{\partial v_{A}}-\frac{\partial h_{1}(0)}{\partial v_{A}}\right)_{L^{2}(\Gamma)} \\
& +\left(z_{2 d}-\frac{\partial h_{2}(0)}{\partial v_{A}}, \frac{\partial h_{2}(v)}{\partial v_{A}}-\frac{\partial h_{2}(0)}{\partial v_{A}}\right)_{L^{2}(\Gamma)} .
\end{aligned}
$$

Then

$$
J(v)=\pi(v, v)-2 f(v)+\left\|z_{1 d}-\frac{\partial h_{1}(0)}{\partial v_{A}}\right\|_{L^{2}(\Gamma)}^{2}+\left\|z_{2 d}-\frac{\partial h_{2}(0)}{\partial v_{A}}\right\|_{L^{2}(\Gamma)}^{2} .
$$

From (15), $\pi(v, v) \geq M\|v\|_{\left(L^{2}(\Gamma)\right)^{2}}^{2}$. Using the theory of Lions [1], there exists a unique optimal control of problem (16), moreover it is characterized by

Theorem 2.2 Let us suppose that (10) holds and the cost functional is given 
by (14), then the boundary control $u$ is characterized by

$$
\begin{aligned}
& -\Delta p_{1}(u)-a p_{1}(u)-c p_{2}(u)=0 \quad \text { in } \Omega, \\
& -\Delta p_{2}(u)-b p_{1}(u)-d p_{2}(u)=0 \quad \text { in } \Omega \text {, } \\
& p_{1}(u)=-\left(\frac{\partial h_{1}(u)}{\partial v_{A}}-z_{1 d}\right), p_{2}(u)=-\left(\frac{\partial h_{2}(u)}{\partial v_{A}}-z_{2 d}\right) \quad \text { on } \Gamma \text {, } \\
& {\left[\sum_{i, j}^{n} \frac{\partial p_{1}(u)}{\partial x_{j}} \cos \left(v, x_{i}\right)\right]=\left[\sum_{i, j}^{n} \frac{\partial p_{2}(u)}{\partial x_{j}} \cos \left(v, x_{i}\right)\right]=0 \quad \text { on } \gamma \text {, }} \\
& \left\{\frac{\partial p_{1}(u)}{\partial v_{A}}\right\}^{ \pm}=\frac{1}{R_{1}+R_{2}}\left[p_{1}(u)\right],\left\{\frac{\partial p_{2}(u)}{\partial v_{A}}\right\}^{ \pm}=\frac{1}{R_{1}+R_{2}}\left[p_{2}(u)\right] \text { on } \gamma \text {, } \\
& \left(p_{1}(u), \frac{\partial h_{1}(v-u)}{\partial v_{A}}-\frac{\partial h_{1}(0)}{\partial v_{A}}\right)_{L^{2}(\Gamma)} \\
& +\left(p_{2}(u), \frac{\partial h_{2}(v-u)}{\partial v_{A}}-\frac{\partial h_{2}(0)}{\partial v_{A}}\right)_{L^{2}(\Gamma)}+(N u, v-u)_{\left(L^{2}(\Gamma)\right)^{2}} \geq 0,
\end{aligned}
$$

together with(11), (12) and (13), where $p(u)=\left\{p_{1}(u), p_{2}(u)\right\}$ is the adjoint state.

Proof.

The optimal control $u$ is characterized by [1]

$$
\pi(u, v-u)-L(v-u) \geq 0 \quad \forall u \in U_{a d} .
$$

Then

$$
\begin{aligned}
& \left(\frac{\partial h_{1}(u)}{\partial v_{A}}-\frac{\partial h_{1}(0)}{\partial v_{A}}, \frac{\partial h_{1}(v-u)}{\partial v_{A}}-\frac{\partial h_{1}(0)}{\partial v_{A}}\right)_{L^{2}(\Gamma)} \\
& +\left(\frac{\partial h_{2}(u)}{\partial v_{A}}-\frac{\partial h_{2}(0)}{\partial v_{A}}, \frac{\partial h_{2}(v-u)}{\partial v_{A}}-\frac{\partial h_{2}(0)}{\partial v_{A}}\right)_{L^{2}(\Gamma)}+(N u, v-u)_{\left(L^{2}(\Gamma)\right)^{2}} \\
& -\left(z_{1 d}-\frac{\partial h_{1}(0)}{\partial v_{A}}, \frac{\partial h_{1}(v-u)}{\partial v_{A}}-\frac{\partial h_{1}(0)}{\partial v_{A}}\right)_{L^{2}(\Gamma)} \\
& -\left(z_{2 d}-\frac{\partial h_{2}(0)}{\partial v_{A}}, \frac{\partial h_{2}(v-u)}{\partial v_{A}}-\frac{\partial h_{2}(0)}{\partial v_{A}}\right)_{L^{2}(\Gamma)} \geq 0 .
\end{aligned}
$$

So

$$
\begin{aligned}
& \left(\frac{\partial h_{1}(u)}{\partial v_{A}}-z_{1 d}, \frac{\partial h_{1}(v-u)}{\partial v_{A}}-\frac{\partial h_{1}(0)}{\partial v_{A}}\right)_{L^{2}(\Gamma)} \\
& +\left(\frac{\partial h_{2}(u)}{\partial v_{A}}-z_{2 d}, \frac{\partial h_{2}(v-u)}{\partial v_{A}}-\frac{\partial h_{2}(0)}{\partial v_{A}}\right)_{L^{2}(\Gamma)}+(N u, v-u)_{\left(L^{2}(\Gamma)\right)^{2}} \geq 0 .
\end{aligned}
$$

Since the model A of the system is given by

$$
A h(x)=A\left(h_{1}, h_{2}\right)=\left(-\Delta h_{1}-a h_{1}-b h_{2},-\Delta h_{2}-c h_{1}-d h_{2}\right),
$$

and since

$$
\left(A^{*} p, h\right)=(p, A h),
$$


then

$$
\begin{aligned}
& (p(u), A h(u)) \\
& =\left(p_{1}(u),-\Delta h_{1}(u)-a h_{1}(u)-b h_{2}(u)\right)+\left(p_{2}(u),-\Delta h_{2}(u)-c h_{1}(u)-d h_{2}(u)\right) \\
& =\left(-\Delta p_{1}(u)-a p_{1}(u)-c p_{2}(u), h_{1}(u)\right)+\left(-\Delta p_{2}(u)-b p_{1}(u)-d p_{2}(u), h_{2}(u)\right)
\end{aligned}
$$

and since the adjoint state is defined by:

$$
\left\{\begin{array}{lc}
-\Delta p_{1}(u)-a p_{1}(u)-c p_{2}(u)=0 & \text { in } \Omega, \\
-\Delta p_{2}(u)-b p_{1}(u)-d p_{2}(u)=0 & \text { in } \Omega, \\
p_{1}(u)=-\left(\frac{\partial h_{1}(u)}{\partial v_{A}}-z_{1 d}\right) & \text { on } \Gamma, \\
p_{2}(u)=-\left(\frac{\partial h_{2}(u)}{\partial v_{A}}-z_{2 d}\right) & \text { on } \Gamma, \\
{\left[\frac{\partial p_{1}(u)}{\partial v_{A^{*}}}\right]^{-}\left[\frac{\partial p_{2}(u)}{\partial v_{A^{*}}}\right]=0} & \text { on } \gamma, \\
\left\{\frac{\partial p_{1}(u)}{\partial v_{A^{*}}}\right\}^{ \pm}=\frac{1}{R_{1}+R_{2}}\left[p_{1}(u)\right],\left\{\frac{\partial p_{2}(u)}{\partial v_{A^{*}}}\right\}^{ \pm}=\frac{1}{R_{1}+R_{2}}\left[p_{2}(u)\right] & \text { on } \gamma,
\end{array}\right.
$$

hence (17) implies

$$
\begin{aligned}
& \left(p_{1}(u), \frac{\partial h_{1}(v-u)}{\partial v_{A}}-\frac{\partial h_{1}(0)}{\partial v_{A}}\right)_{L^{2}(\Gamma)} \\
& +\left(p_{2}(u), \frac{\partial h_{2}(v-u)}{\partial v_{A}}-\frac{\partial h_{2}(0)}{\partial v_{A}}\right)_{L^{2}(\Gamma)}+(N u, v-u)_{\left(L^{2}(\Gamma)\right)^{2}} \geq 0 .
\end{aligned}
$$

\section{Boundary Control for $2 \times 2$ Neumann Elliptic Systems}

In this section, we study the boundary control for $2 \times 2$ cooperative Neumann elliptic system in the form

$$
\begin{cases}-\Delta h_{1}=a h_{1}+b h_{1}+f_{1} & \text { in } \Omega \subset R^{n}, \\ -\Delta h_{2}=c h_{1}+d h_{2}+f_{2} & \text { in } \Omega \subset R^{n}, \\ \frac{\partial h_{1}}{\partial v_{A}}=g_{1}, \frac{\partial h_{2}}{\partial v_{A}}=g_{2} & \text { on } \Gamma,\end{cases}
$$

with conjugation conditions (4) and (5), where $g_{i} \in\left(L^{2}(\Gamma)\right)^{2}, i=1,2$. For this, we introduce again the bilinear form (9) which is coercive on $\left(H^{1}(\Omega)\right)^{2}$, since

$$
\left(H_{0}^{1}(\Omega)\right)^{2} \subseteq\left(H^{1}(\Omega)\right)^{2} .
$$

Then, by Lax-Milgram lemma, there exists a unique solution $h=\left(h_{1}, h_{2}\right)$ for system (18) such that:

$$
a(h, \psi)=L(\psi) \quad \forall \psi \in\left(H^{1}(\Omega)\right)^{2},
$$

where 


$$
\begin{aligned}
L_{g}(\psi)= & \int_{\Omega} f_{1}(x) \psi_{1}(x) \mathrm{d} x+\int_{\Omega} f_{2}(x) \psi_{2}(x) \mathrm{d} x \\
& +\int_{\Gamma} g_{1}(x) \psi_{1}(x) \mathrm{d} \Gamma+\int_{\Gamma} g_{2}(x) \psi_{2}(x) \mathrm{d} \Gamma+\int_{\gamma} \frac{\left(R_{2} w_{1}-\delta\right)\left[\psi_{1}\right]}{R_{1}+R_{2}} \mathrm{~d} \gamma \\
& +\int_{\gamma} \frac{\left(R_{2} w_{2}-\delta\right)\left[\psi_{2}\right]}{R_{1}+R_{2}} \mathrm{~d} \gamma-\int_{\gamma} w_{1} \psi_{1}^{+} \mathrm{d} \gamma-\int_{\gamma} w_{2} \psi_{2}^{+} \mathrm{d} \gamma,
\end{aligned}
$$

is a continuous linear form defined on $\left(H^{1}(\Omega)\right)^{2}$. Then, applying Green's formula, we get

$$
\begin{aligned}
& \int_{\Omega}\left(-\Delta h_{1}-a h_{1}-b h_{2}\right) \psi_{1}(x) \mathrm{d} x+\int_{\Omega}\left(-\Delta h_{2}-c h_{1}-d h_{2}\right) \psi_{2}(x) \mathrm{d} x \\
& =-\int_{\Gamma} \frac{\partial h_{1}}{\partial v_{A}} \psi_{1}(x) \mathrm{d} \Gamma-\int_{\Gamma} \frac{\partial h_{2}}{\partial v_{A}} \psi_{2}(x) \mathrm{d} \Gamma-\int_{\gamma} \frac{\partial h_{1}}{\partial v_{A}} \psi_{1}(x) \mathrm{d} \gamma \\
& -\int_{\gamma} \frac{\partial h_{2}}{\partial v_{A}} \psi_{2}(x) \mathrm{d} \gamma+a(h, \psi)=\int_{\Omega} f_{1} \psi_{1} \mathrm{~d} x+\int_{\Omega} f_{2} \psi_{2} \mathrm{~d} x,
\end{aligned}
$$

using (19), the state $h(u)=\left(h_{1}(u), h_{2}(u)\right)$ of the system is given by the solution of

$$
\begin{cases}-\Delta h_{1}(u)=a h_{1}(u)+b h_{2}(u)+f_{1} & \text { in } \Omega=\Omega_{1} \cup \Omega_{2}, \\ -\Delta h_{2}(u)=c h_{1}(u)+d h_{2}(u)+f_{2} & \text { in } \Omega=\Omega_{1} \cup \Omega_{2}, \\ \frac{\partial h_{1}(u)}{\partial v_{A}}=g_{1}+u_{1} & \text { on } \Gamma, \\ \frac{\partial h_{2}(u)}{\partial v_{A}}=g_{2}+u_{2} & \text { on } \Gamma,\end{cases}
$$

under conjugation conditions (12), (13). For a given $z_{d}=\left(z_{1 d}, z_{2 d}\right) \in\left(L^{2}(\Gamma)\right)^{2}$, the cost functional is again given by (14), then there exists a unique optimal control $u \in U_{a d}$ for (16) and we deduce:

Theorem 3.1 If the cost functional is given by (14), there exists a unique boundary control

$$
\begin{aligned}
& u=\left(u_{1}, u_{2}\right) \in\left(L^{2}(\Gamma)\right)^{2} \text {, such that: } \\
& \qquad J(u) \leq J(v) \quad \forall v \in U_{a d} \subset\left(L^{2}(\Gamma)\right)^{2},
\end{aligned}
$$

moreover it is characterized by the following equations and inequalities

$$
\begin{cases}-\Delta p_{1}(u)-a p_{1}(u)-c p_{2}(u)=0 & \text { in } \Omega, \\ -\Delta p_{2}(u)-b p_{1}(u)-d p_{2}(u)=0 & \text { in } \Omega, \\ \frac{\partial p_{1}(u)}{\partial v_{A^{*}}}=h_{1}(u)-z_{1 d} & \text { on } \Gamma, \\ \frac{\partial p_{2}(u)}{\partial v_{A^{*}}}=h_{2}(u)-z_{2 d} & \text { on } \Gamma, \\ {\left[\frac{\partial p_{1}(u)}{\partial v_{A^{*}}}\right]=\left[\frac{\partial p_{2}(u)}{\partial v_{A^{*}}}\right]=0} & \text { on } \gamma, \\ \left\{\frac{\partial p_{1}}{\partial v_{A^{*}}}\right\}^{ \pm}=\frac{1}{R_{1}+R_{2}}\left[p_{1}(u)\right],\left\{\frac{\partial p_{2}(u)}{\partial v_{A^{*}}}\right\}^{ \pm}=\frac{1}{R_{1}+R_{2}}\left[p_{2}(u)\right] & \text { on } \gamma,\end{cases}
$$




$$
\int_{\Gamma}\left(p_{1}(u)+N u_{1}\right)\left(v_{1}-u_{1}\right) \mathrm{d} \Gamma+\int_{\Gamma}\left(p_{2}(u)+N u_{2}\right)\left(v_{2}-u_{2}\right) \mathrm{d} \Gamma \geq 0 .
$$

Together with (20), (12) and (13).

\section{Boundary Control for $\boldsymbol{n} \times \boldsymbol{n}$ Cooperative Dirichlet Systems}

In this section, we generalize the discussion which has been introduced in section 2 to $n \times n$ cooperative Dirichlet system of the form

$$
\begin{cases}-\Delta h_{i}+\sum_{j=1}^{n} a_{i j} h_{j}=f_{i} & \text { in } \Omega, \\ h_{i}=0 & \text { on } \Gamma, i=1,2, \cdots, n,\end{cases}
$$

under conjugation conditions

$$
R_{1}\left\{\frac{\partial h_{i}}{\partial v_{A}}\right\}^{-}+R_{2}\left\{\frac{\partial h_{i}}{\partial v_{A}}\right\}^{+}=\left[h_{i}\right]+\delta \quad \text { on } \gamma,
$$

and

$$
\left[\frac{\partial h_{i}}{\partial v_{A}}\right]=\left[\sum_{i, j=1}^{n} \frac{\partial h_{i}}{\partial x_{j}} \cos \left(v, x_{i}\right)\right]=w_{i} \quad \text { on } \gamma, \quad i=1,2, \cdots, n .
$$

To prove the existence of the state of system (21), we assume that:

$$
\left\{\begin{array}{l}
\text { the matrex }(\mu-M I) \text { is a non-singular } M \text {-matrix which means } \\
\text { that all the principal minors extracted from it are positive, }
\end{array}\right.
$$

where, $I$ is identity matrix and $\mu$ is a positive constant determined by Friedrich inequality (8).

By Cartesian product, we have the following chain of Sobolev spaces:

$$
\left(H_{0}^{1}(\Omega)\right)^{n} \subseteq\left(L^{2}(\Omega)\right)^{n} \subseteq\left(H^{-1}(\Omega)\right)^{n} .
$$

On $\left(H_{0}^{1}(\Omega)\right)^{n}$, the bilinear form is defined by:

$$
a(h, \psi)=\sum_{i=1}^{n} \int_{\Omega} \nabla h_{i} \nabla \psi_{i} \mathrm{~d} x+\sum_{i, j=1}^{n} \int_{\Omega} a_{i j} h_{i} \psi_{i} \mathrm{~d} x+\sum_{i=1}^{n} \int_{\gamma} \frac{\left[h_{i}\right]\left[\psi_{i}\right]}{R_{1}+R_{2}} \mathrm{~d} \gamma .
$$

As in lemma 2.1, (24), implies

$$
a(h, h) \geq C \sum_{i=1}^{n}\left\|h_{i}\right\|^{2}=C\|h\|_{\left(H_{0}^{1}(\Omega)\right)^{n}}^{2}, \forall h \in\left(H_{0}^{1}(\Omega)\right)^{n} .
$$

Now, let

$$
L(\psi)=\sum_{i=1}^{n} \int_{\Omega} f_{i}(x) \psi_{i}(x) \mathrm{d} x+\sum_{i=1}^{n} \int_{\gamma} \frac{\left(R_{2} w-\delta\right)\left[\psi_{i}\right]}{R_{1}+R_{2}} \mathrm{~d} \gamma-\sum_{i=1}^{n} \int_{\gamma} w_{i} \psi_{i}^{+} \mathrm{d} \gamma,
$$

be a continuous linear form on $\left(H_{0}^{1}(\Omega)\right)^{n}$, then using Lax-Milgram lemma, there exists a unique solution $h \in\left(H_{0}^{1}(\Omega)\right)^{n}$ such that:

$$
a(h, \psi)=L(\psi) \quad \forall \psi=\left(\psi_{i}\right)_{i=1}^{n} \in\left(H_{0}^{1}(\Omega)\right)^{n} .
$$

Then, we have

Theorem 4.1 For $f=\left\{f_{i}\right\}_{i=1}^{n} \in\left(L^{2}(\Omega)\right)^{n}$ there exists a unique solution 
$h \in\left(H_{0}^{1}(\Omega)\right)^{n}$ for cooperative Dirichlet system (21) with conjugation conditions (22) and (23) if condition (24) is satisfied.

So, we can formulate the corresponding control problem:

The space $U=\left(L^{2}(\Gamma)\right)^{n}$ is the space of controls. For a control $u=\left\{u_{1}, u_{2}, \cdots, u_{n}\right\} \in\left(L^{2}(\Gamma)\right)^{n}$, the state $h(u)=\left\{h_{1}(u), h_{2}(u), \cdots, h_{n}(u)\right\}$ of the system is given by the solution of

$$
\begin{cases}-\Delta h_{i}(u)+\sum_{j=1}^{n} a_{i j} h_{j}(u)=f_{i}(u) & \text { in } \Omega, \\ h_{i}(u)=u_{i} & \text { on } \Gamma, i=1,2, \cdots, n,\end{cases}
$$

under conjugation conditions:

$$
\begin{cases}R_{1}\left\{\frac{\partial h_{i}(u)}{\partial v_{A}}\right\}^{-}+R_{2}\left\{\frac{\partial h_{i}(u)}{\partial v_{A}}\right\}^{+}=\left[h_{i}(u)\right]+\delta & \text { on } \gamma, \\ {\left[\frac{\partial h_{i}(u)}{\partial v_{A}}\right]=\left[\sum_{i, j=1}^{n} \frac{\partial h_{i}(u)}{\partial x_{j}} \cos \left(v, x_{i}\right)\right]=w_{i}} & \text { on } \gamma, i=1,2, \cdots, n .\end{cases}
$$

The observation may be takes as

$$
\begin{aligned}
z(u) & =\left\{z_{1}(u), z_{2}(u), \cdots, z_{n}(u)\right\}=C h(u)=C\left\{h_{1}(u), h_{2}(u), \cdots, h_{n}(u)\right\} \\
& =\left\{h_{1}(u), h_{2}(u), \cdots, h_{n}(u)\right\},
\end{aligned}
$$

the cost functional is given by

$$
J(v)=\sum_{i=1}^{n}\left\|\frac{\partial h_{i}(v)}{\partial v_{A}}-z_{i d}\right\|_{L^{2}(\Gamma)}^{2}+(N v, v)_{\left(L^{2}(\Gamma)\right)^{n}},
$$

where $N$ is a hermitian positive definite operator such that:

$$
(N v, v)_{\left(L^{2}(\Gamma)\right)^{n}} \geq M\|v\|_{\left(L^{2}(\Gamma)\right)^{n}}^{2}, \quad M>0, \quad \forall v \in U .
$$

The control problem then is to find:

$$
\left\{\begin{array}{l}
u=\left\{u_{1}, u_{2}, \cdots, u_{n}\right\} \in U_{a d} \text { such that : } \\
J(u)=\inf J(v) \quad \forall v \in U_{a d},
\end{array}\right.
$$

where $U_{a d}$ is closed convex subset of $\left(L^{2}(\Gamma)\right)^{n}$. The cost functional (30) can be written as

$$
J(v)=\sum_{i=1}^{n}\left\|\frac{\partial h_{i}(v)}{\partial v_{A}}-\frac{\partial h_{i}(0)}{\partial v_{A}}+\frac{\partial h_{i}(0)}{\partial v_{A}}-z_{i d}\right\|_{L^{2}(\Gamma)}^{2}+(N v, v)_{\left(L^{2}(\Gamma)\right)^{n}},
$$

if we let

$$
\pi(u, v)=\sum_{i=1}^{n}\left(\frac{\partial h_{i}(u)}{\partial v_{A}}-\frac{\partial h_{i}(0)}{\partial v_{A}}, \frac{\partial h_{i}(v)}{\partial v_{A}}-\frac{\partial h_{i}(0)}{\partial v_{A}}\right)_{L^{2}(\Gamma)}+(N v, v)_{\left(L^{2}(\Gamma)\right)^{n}},
$$

and

$$
L(v)=\sum_{i=1}^{n}\left(z_{i d}-\frac{\partial h_{i}(0)}{\partial v_{A}}, \frac{\partial h_{i}(v)}{\partial v_{A}}-\frac{\partial h_{i}(0)}{\partial v_{A}}\right)_{L^{2}(\Gamma)},
$$


then

$$
J(v)=\pi(v, v)-2 L(v)+\sum_{i=1}^{n}\left\|z_{i d}-\frac{\partial h_{i}(0)}{\partial v_{A}}\right\|_{L^{2}(\Gamma)}^{2} .
$$

From (31),

$$
\pi(v, v) \geq N\|v\|_{\left(L^{2}(\Gamma)\right)^{n}}^{2},
$$

using the theory of Lions [1], there exists a unique optimal control of problem(32); moreover it is characterized by

Theorem 4.2 Let us suppose that (26) holds and the cost functional is given by(13), then the boundary control $u$ is characterized by

$$
\begin{cases}-\Delta p_{i}(u)+\sum_{j=1}^{n} a_{i j} p_{i}(u)=0 & \text { in } \Omega, \\ p_{i}(u)=-\left(\frac{\partial h_{i}(u)}{\partial v_{A}}-z_{i d}\right) & \text { on } \Gamma, \\ {\left[\frac{\partial p_{i}(u)}{\partial v_{A^{*}}}\right]=0,} & \text { on } \gamma, \\ \left\{\frac{\partial p_{i}(u)}{\partial v_{A^{*}}}\right\}^{ \pm}=\frac{1}{R_{1}+R_{2}}\left[p_{i}(u)\right], & \text { on } \gamma, \\ \sum_{i=1}^{n} \int_{\Gamma} \frac{\partial p_{i}(u)}{\partial v_{A^{*}}} N u_{i}\left(v_{i}-u_{i}\right) \mathrm{d} \Gamma \geq 0, & \end{cases}
$$

together with (28) and (29) where $p(u)=\left\{p_{1}(u), p_{2}(u), \cdots, p_{n}(u)\right\}$ is the adjoint state.

Proof. The optimal control $u=\left\{u_{i}\right\}_{i=1}^{n} \in\left(L^{2}(\Gamma)\right)^{n}$ is characterized by:

$$
\begin{aligned}
& \pi(u, v-u) \geq L(v-u) \quad \forall v=\left\{v_{1}, v_{2}, \cdots, v_{n}\right\} \in U_{a d}, \\
& \pi(u, v-u)-L(v-u) \\
& =\sum_{i=1}^{n}\left(\frac{\partial h_{i}(u)}{\partial v_{A}}-\frac{\partial h_{i}(0)}{\partial v_{A}}, \frac{\partial h_{i}(v-u)}{\partial v_{A}}-\frac{\partial h_{i}(0)}{\partial v_{A}}\right)_{L^{2}(\Gamma)} \\
& +\sum_{i=1}^{n}\left(N u_{i}, v_{i}-u_{i}\right)_{L^{2}(\Gamma)}-\left(z_{i d}-\frac{\partial h_{i}(0)}{\partial v_{A}}, \frac{\partial h_{i}(v-u)}{\partial v_{A}}-\frac{\partial h_{i}(0)}{\partial v_{A}}\right)_{L^{2}\left(L^{2}(\Gamma)\right)} \\
& \geq 0,
\end{aligned}
$$

then

$$
\sum_{i=1}^{n}\left(\frac{\partial h_{i}(u)}{\partial v_{A}}-z_{i d}, \frac{\partial h_{i}(v-u)}{\partial v_{A}}-\frac{\partial h_{i}(0)}{\partial v_{A}}\right)_{L^{2}(\Gamma)}+\sum_{i=1}^{n}\left(N u_{i}, v_{i}-u_{i}\right)_{L^{2}(\Gamma)} \geq 0,
$$

since the adjoint state is defined by (33), (34) implies

$$
\sum_{i=1}^{n}\left(p_{i}(u), \frac{\partial h_{i}(v-u)}{\partial v_{A}}-\frac{\partial h_{i}(0)}{\partial v_{A}}\right)_{L^{2}(\Gamma)}+\sum_{i=1}^{n}\left(N u_{i}, v_{i}-u_{i}\right)_{L^{2}(\Gamma)} \geq 0 .
$$

Applying Green's formula, we obtain 


$$
\begin{aligned}
& \sum_{i=1}^{n}\left(A^{*} p_{i}(u), h_{i}(v-u)\right)_{L^{2}(\Omega)}+\sum_{i=1}^{n}\left(\frac{\partial p_{i}(u)}{\partial v_{A^{*}}}, h_{i}(v-u)\right)_{L^{2}(\Gamma)} \\
& +a(p(u), h(v-u))+\sum_{i=1}^{n}\left(N u_{i}, v_{i}-u_{i}\right)_{L^{2}(\Gamma)} \geq 0 .
\end{aligned}
$$

Since

$$
(p, A h)=\left(A^{*} p, h\right)=a(p, h) \text { and } A^{*} p=0,
$$

we obtain by using equation (28),

$$
\sum_{i=1}^{n}\left(\frac{\partial p_{i}(u)}{\partial v_{A^{*}}}, v_{i}-u_{i}\right)_{L^{2}(\Gamma)}+\sum_{i=1}^{n}\left(N u_{i}, v_{i}-u_{i}\right)_{L^{2}(\Gamma)} \geq 0,
$$

hence

$$
\sum_{i=1}^{n} \int_{\Gamma} \frac{\partial p_{i}(u)}{\partial v_{A^{*}}} N u_{i}\left(v_{i}-u_{i}\right) \mathrm{d} \Gamma \geq 0
$$

\section{Boundary Control for $n \times n$ Cooperative Neumann Systems}

We generalize here, the results which have been established in section (3) to the following $n \times n$ Neumann elliptic system

$$
\begin{cases}-\Delta h_{i}+\sum_{j=1}^{n} a_{i j} h_{j}=f_{i} & \text { in } \Omega, \\ \frac{\partial h_{i}}{\partial v_{A}}=g_{i} & \text { on } \Gamma, i=1,2, \cdots, n,\end{cases}
$$

with conjugation conditions (22) and (23), where $g=\left\{g_{1}, g_{2}, \cdots, g_{n}\right\} \in\left(L^{2}(\Gamma)\right)^{n}$ are given functions. Since

$$
\left(H_{0}^{1}(\Omega)\right)^{n} \subseteq\left(H^{1}(\Omega)\right)^{n},
$$

the bilinear form (25) is coercive on $\left(H^{1}(\Omega)\right)^{n}$.

Then using Lax-Milgram lemma, there exists a unique solution $y$ for system (35) such that:

$$
a(h, \psi)=L_{N}(\psi), \quad \forall \psi \in\left(H^{1}(\Omega)\right)^{n},
$$

where

$$
\begin{aligned}
L_{N}(\psi)= & \sum_{i=1}^{n} \int_{\Omega} f_{i}(x) \psi_{i}(x) \mathrm{d} x+\sum_{i=1}^{n} \int_{\Gamma} g_{i}(x) \psi_{i}(x) \mathrm{d} x \\
& +\sum_{i=1}^{n} \int_{\gamma} \frac{\left(R_{2} w-\delta\right)\left[\psi_{i}\right]}{R_{1}+R_{2}} \mathrm{~d} \gamma-\sum_{i=1}^{n} \int_{\gamma} w \psi_{i}^{+} \mathrm{d} \gamma,
\end{aligned}
$$

is a continuous linear form defined on $\left(H^{1}(\Omega)\right)^{n}$. Let us multiply both sides of first equation of (35) by $\psi \in\left(H^{1}(\Omega)\right)^{n}$ and integrate over $\Omega$, we get

$$
\sum_{i=1}^{n} \int_{\Omega}\left(-\Delta h_{i}+\sum_{j=1}^{n} a_{i j} h_{j}\right) \psi_{i}(x) \mathrm{d} x=\sum_{i=1}^{n} \int_{\Omega} f_{i} \psi_{i} \mathrm{~d} x
$$

Applying Green's formula, 


$$
\begin{aligned}
& \quad \sum_{i=1}^{n} \int_{\Omega}\left(-\Delta h_{i}\right) \psi_{i} \mathrm{~d} x-\sum_{i=1}^{n} \int_{\Omega} a_{i j} h_{i} \psi_{i} \mathrm{~d} x-\sum_{i=1}^{n} \int_{\Gamma} \frac{\partial h_{i}}{\partial v_{A}} \psi_{i} \mathrm{~d} \Gamma \\
& -\sum_{i=1}^{n} \int_{\gamma} \frac{\partial h_{i}}{\partial v_{A}} \psi_{i} \mathrm{~d} \gamma+\sum_{i=1}^{n} \int_{\gamma} \frac{\left[h_{i}\right]\left[\psi_{i}\right]}{R_{1}+R_{2}} \mathrm{~d} \gamma \\
& \quad=\sum_{i=1}^{n} \int_{\Gamma} g_{i} \psi_{i} \mathrm{~d} \Gamma+\sum_{i=1}^{n} \int_{\Omega} f_{i} \psi_{i} \mathrm{~d} x \\
& \sum_{i=1}^{n} \int_{\Omega}\left(-\Delta h_{i}-\sum_{j=1}^{n} a_{i j} h_{j}\right) \psi_{i}(x) \mathrm{d} x-\sum_{i=1}^{n} \int_{\Gamma}\left(\frac{\partial h_{i}}{\partial v_{A}}\right) \psi_{i}(x) \mathrm{d} \Gamma \\
& -\sum_{i=1}^{n} \int_{\gamma}\left(\frac{\partial h_{i}}{\partial v_{A}}\right) \psi_{i}(x) \mathrm{d} \gamma+\sum_{i=1}^{n} \int_{\gamma} \frac{\left[h_{i}\right]\left[\psi_{i}\right]}{R_{1}+R_{2}} \mathrm{~d} \gamma \\
& =\sum_{i=1}^{n} \int_{\Gamma} g_{i} \psi_{i} \mathrm{~d} \Gamma+\sum_{i=1}^{n} \int_{\Omega} f_{i} \psi_{i} \mathrm{~d} x,
\end{aligned}
$$

then from

$$
a(h, \psi)=L_{N}(\psi),
$$

we obtain the Neumann conditions

$$
\frac{\partial h_{i}}{\partial v_{A}}=g_{i} \quad \text { on } \Gamma \text {. }
$$

Then, we have the corresponding control problem:

The space $U=\left(L^{2}(\Gamma)\right)^{n}$ is the space of controls, the state $h(u)=\left\{h_{1}(u), h_{2}(u), \cdots, h_{n}(u)\right\}$ of the system is given by the solution of

$$
\begin{cases}-\Delta h_{i}(u)+\sum_{j=1}^{n} a_{i j} h_{j}(u)=f_{i}(u) & \text { in } \Omega, \\ \frac{\partial h_{i}(u)}{\partial v_{A}}=g_{i}+u_{i} & \text { on } \Gamma,\end{cases}
$$

under conjugation conditions (29), where $u=\left\{u_{1}, u_{2}, \cdots, u_{n}\right\}$ is a given control in $\left(L^{2}(\Gamma)\right)^{n}$. For a given $z_{d}=\left\{z_{1 d}, z_{2 d}, \cdots, z_{n d}\right\} \in\left(L^{2}(\Gamma)\right)^{n}$, the cost functional is again given by (30). As in theorem (4.2), we can prove:

Theorem 5.1 Let us suppose that (26) holds and the cost functional is given by (30), there exists a unique optimal control $u$, such that.

$$
J(u)=\inf J(v) \quad \forall v \in U_{a d} \subset\left(L^{2}(\Gamma)\right)^{n},
$$

moreover it is characterized by the following equations and inequalities

$$
\begin{cases}-\Delta p_{i}(u)+\sum_{j=1}^{n} a_{i j} p_{i}(u)=0 & \text { in } \Omega, \\ \frac{\partial p_{i}(u)}{\partial v_{A^{*}}}=\left(\frac{\partial h_{i}(u)}{\partial v_{A}}-z_{i d}\right) & \text { on } \Gamma, \\ {\left[\frac{\partial p_{i}(u)}{\partial v_{A^{*}}}\right]^{\prime}=0} & \text { on } \gamma, \\ \left\{\frac{\partial p_{i}(u)}{\partial v_{A^{*}}}\right\}^{ \pm}=\frac{1}{R_{1}+R_{2}}\left[p_{i}(u)\right] & \text { on } \gamma,\end{cases}
$$




$$
\sum_{i=1}^{n}\left(p_{i}(u), v_{i}-u_{i}\right)_{\left(L^{2}(\Gamma)\right)^{n}}+(N u, v-u)_{\left(L^{2}(\Gamma)\right)^{n}} \geq 0, \quad i=1,2, \cdots, n,
$$

together with(36) and (29), where $p(u)=\left\{p_{1}(u), p_{2}(u), \cdots, p_{n}(u)\right\}$ is the adjoint state.

\section{Conclusion}

In the present work, we concentrated on optimal control problems for cooperative elliptic systems under conjugation conditions. We proved the existence and uniqueness of the state for $2 \times 2$ Dirichlet cooperative elliptic system. Then we discussed the existence and uniqueness of the optimal control of boundary type for this system and we gave the set of equations and inequalities that characterizes this control. Also, we studied the problem with Neumann condition. At last, we generalized the discussion to $n \times n$ cooperative elliptic systems under conjugation conditions.

\section{Acknowledgements}

The authors would like to express their gratitude to Professor Dr. I. M. Gali, Mathematics Department, Faculty of Science, Al-Azhar University, for suggesting the problem and critically reading the manuscript.

\section{Conflicts of Interest}

The authors declare no conflicts of interest regarding the publication of this paper.

\section{References}

[1] Lions, J.L. (1971) Optimal Control of a System Governed by Partial Differential Equations. Springer-Verlag, New York, 170. https://doi.org/10.1007/978-3-642-65024-6

[2] Kotarski, W. and El-Saify, H.A. (2006) Optimality of the Boundary Control Problem for $n \times n$ Parabolic Lag System. Journal of Mathematical Analysis and Applications, 319, 61-73.

[3] Serag, H.M. and Qamlo, A.H. (2001) Boundary Control of Non-Cooperative Elliptic System. Advances in Modeling Analysis, 38, 31-42.

[4] El-Saify, H.A., Serag, H.M. and Shehata, M.A. (2009) Time Optimal Control Problem for Cooperative Hyperbolic Systems Involving the Laplace Operator. Journal of Dynamical and Control Systems, 15, 405-423. https://doi.org/10.1007/s10883-009-9067-y

[5] Gali, I.M. and Serag, H.M. (1995) Optimal Control of Cooperative Elliptic Systems Defined on $R^{n}$. Journal of the Egyptian Mathematical Society, 3, 33-39.

[6] Serag, H.M. (2007) Distributed Control for Cooperative Systems Involving Parabolic Operators with an Infinite Number of Variables. The IMA Journal of Mathematical Control and Information, 24, 149-161. https://doi.org/10.1093/imamci/dnl018

[7] Serag, H.M., EL-Zahaby, S.A. and Abd Elrhman, L.M. (2015) Distributed Control for Cooperative Parabolic Systems with Conjugation Conditions. Journal of Progressive Research in Mathematics, 4, 348-365. 
[8] Qamlo, A.H. and Bedaiwi, G.M. (2017) Distributed Control for $2 \times 2$ Coupled Infinite Order Hyperbolic Systems. Advances in Differential Equations and Control Processes, 18, 201-227. https://doi.org/10.17654/DE018040201

[9] Qamlo, A.H. (2021) Boundary Control Problems for $2 \times 2$ Cooperative Hyperbolic Systems with Infinite Order Operators. Open Journal of Optimization, 10, 1-12. https://doi.org/10.4236/ojop.2021.101001

[10] El-Saify, H.A. and Serag, H.M. (1991) Boundary Control of a Systems Governed by Non-Linear Neumann Problem. Journal of Institute of Mathematics \& Computer Sciences, 2, 41-48.

[11] Khafagy, S. and Serag, H.M. (2018) Stability Results of Positive Weak Solution for Singular p-Laplacian Nonlinear System. Journal of Applied Mathematics \& Informatics, 36, 173-179.

[12] Khafagy, S. and Serag, H.M. (2020) On the Existence of Positive Weak Solution for Nonlinear System with Singular Weights. Journal of Contemporary Mathematical Analysis (Armenian Academy of Sciences), 55, 259-267.

[13] Serag, H.M. and Khafagy, S. (2008) Existence of Weak Solution for $n \times n$ Nonlinear Systems Involving Different Degenerated p-Laplacian Operators. New Zealand Journal of Mathematics, 38, 75-86.

[14] Serag, H.M. and Khafagy, S. (2010) On Maximum Principle and Existence of Positive Weak Solutions for $n \times n$ Nonlinear Elliptic Systems Involving Degenerated p-Laplacian Operators. Turkish Journal of Mathematics, 34, 59-72.

[15] Hassan, H.M. and Serag, H.M. (2000) Boundary Control of Quasi-Static Problem with Viscous Boundary Conditions. Indian Journal of Pure and Applied Mathematics, 31, 767-772.

[16] Sergienko, I.V. and Deineka, V.S. (2001) The Dirichlet and Neumann Problems for Elliptical Equations with Conjugation Conditions and High-Precision Algorithms of Their Discretization. Cybernetics and Systems Analysis, 37, 323-347. https://doi.org/10.1023/A:1011933625923

[17] Sergienko, I.V. and Deineka, V.S. (2004) Optimal Control of an Elliptic System with Conjugation Conditions and Neumann Boundary Conditions. Cybernetics and Systems Analysis, 40, 865-882. https://doi.org/10.1007/s10559-005-0026-7

[18] Sergienko, I.V. and Deineka, V.S. (2005) Optimal Control of Distributed Systems with Conjugation Conditions. Springer, Berlin.

[19] Fleckinger, J. and Serag, H.M. (1995) Semilinear Cooperative Elliptic Systems on $R^{n}$. Rendiconti di Matematica e delle sue Applicazioni, 15, 98-108. 